\title{
Conventional Amphotericin B Associated Nephrotoxicity in Patients With Hematologic Malignancies
}

Vildan Gursoy ${ }^{1}$, Fahir Ozkalemkas ${ }^{2}$, Vildan Ozkocaman ${ }^{2}$, Zafer Serenli Yegen ${ }^{3}$, Ibrahim Ethem Pinar ${ }^{2}$, Beyza Ener ${ }^{4}$, Halis Akalın ${ }^{5}$, Esra Kazak ${ }^{5}$, Ridvan Ali $^{2}$, Alparslan Ersoy 6

1. Division of Hematology, Department of Internal Medicine, Medical School of Usak University, Usak, TUR 2. Division of Hematology, Department of Internal Medicine, Uludag University Medical Faculty, Bursa, TUR 3. Division of Hematology, Bursa City Hospital, Bursa, TUR 4. Department of Microbiology, Uludag University Medical Faculty, Bursa, TUR 5. Department of Infectious Diseases and Clinical Microbiology, Uludag University Medical Faculty, Bursa, TUR 6. Division of Nephrology, Department of Internal Medicine, Uludag University Medical Faculty, Bursa, TUR

Corresponding author: Vildan Gursoy, vildanterzioglu@hotmail.com

\begin{abstract}
Introduction: Amphotericin B (AmB-d) is one of the most effective therapeutic options against frequently life-threatening systemic fungal infections in patients with hematologic malignancies. However, significant adverse effects including nephrotoxicity associated with its use limit its more widespread use. The objectives of our study were to determine the incidence of AmB-d associated nephrotoxicity, to evaluate clinical and epidemiological characteristics of patients, and to support the notion that conventional amphotericin B remains a valid therapeutic option among hematologic patients with proper patient selection.
\end{abstract}

Materials and methods: A total of 110 patients with hematologic malignancies were admitted to our Hematology Unit between January 2014 and November 2017 who required anti-fungal therapy during intensive systemic chemotherapy. The incidence of AmB-d associated nephrotoxicity, side effect profile, time to nephrotoxicity, and clinical and epidemiological characteristics associated with treatment success were assessed retrospectively.

Results: Of the 110 patients receiving AmB-d, 70 (63.6\%) were male and 40 (36.4\%) were female. The mean age of participants was 44 years. The most common diagnosis was acute myeloid leukemia $(n=53,48.2 \%)$, and the most common chemotherapy protocol was $7+3$ remission-induction (cytarabine $100 \mathrm{mg} / \mathrm{m}^{2}$ days 1 7, Idarubicin $12 \mathrm{mg} / \mathrm{m}^{2}$ days $1-3 ; \mathrm{n}=24,21.8 \%$ ). In $56.4 \%$ of the patients, antifungal therapy was given empirically. In 40 patients (36.4\%), nephrotoxicity was observed following antifungal treatment, and only four patients had stage 3 renal failure. The mean duration of time to nephrotoxicity from initiation of amphotericin B was four days (min: 2, max: 31). All patients were found to receive at least one additional potential nephrotoxic treatment during the antifungal treatment process.

Review began 06/16/2021 Review ended 07/06/2021 Published 07/17/2021

() Copyright 2021 Gursoy et al. This is an open access article distributed under the terms of the Creative Commons Attribution License CC-BY 4.0., which permits unrestricted use, distribution, and reproduction in any medium, provided the original author and source are credited.
Conclusion: AmB-d is associated with a significant risk of nephrotoxicity. In most hematological patients, antifungal treatment is initiated empirically, and patients received prolonged courses of treatment. Therefore, it is plausible to initiate such treatment with AmB-d, when one considers the already high treatment costs in this patient group as well as the fact that AmB-d offers similar efficacy to antifungal agents at a lower cost. AmB-d may be recommended as a first-line agent in this patient group with the introduction of newer and more costly antifungal agents when needed, on the basis of the fact that these patients can be closely monitored in a hospital setting, reversible nature of nephrotoxicity upon discontinuation, and rare occurrence of severe renal failure requiring dialysis.

Categories: Infectious Disease, Nephrology, Hematology

Keywords: conventional amphotericin b, nephrotoxicity, hematological malignancy, hypokalemia, fungal infections

\section{Introduction}

Despite recent therapeutic advances, severe fungal infections remain an important cause of morbidity and mortality in hematology units. The traditional amphotericin B was described in the mid-1950s as the first anti-fungal agent effective against systemic mycoses [1]. Amphotericin-B (AmB-d) deoxycholate, the oldest polyene class of drugs, has been approved for use in the treatment of invasive fungal infections and has been considered as a "gold standard" drug since the 1960s [2,3]. The efficacy and safety of newly developed antifungal agents are tested against this agent [4]. AmB-d exhibits a wide spectrum of activity against a number of microorganisms including Aspergillus fumigatus, Blastomyces dermatitidis, Candida spp., Coccidioides immitis, Cryptococcus neoformans, Histoplasma capsulatum, and Paracoccidioides brasiliensis [5]. However, toxicity associated with the use of AmB-d, and particularly nephrotoxicity, is a major factor limiting its clinical utility [6]. Important characteristics of AmB-d associated nephrotoxicity include increased serum creatinine, reduced glomerular filtration rate (GFR), urinary loss of potassium, hypokalemia, urinary loss of magnesium, and hypomagnesemia [7]. 
Due to these toxicities, the role of other antifungal agents as an alternative to AmB- $\mathrm{d}$ has been evaluated for empirical treatment. Lipid formulations have been shown to provide similar efficacy to AmB-d, with a reduced risk of nephrotoxicity [8,9]. However, these formulations are more costly as compared to the conventional forms. Since these preparations generally require higher doses than AmB-d, the cost is even higher. In developing countries such as Turkey, treatment costs are an important consideration when choosing therapeutic agents due to the prolonged hospital stay among patients with hematologic malignancies. A cost-effective treatment with minimal risks may be possible by proper patient selection and management.

In this study, our objectives were to determine AmB-d associated nephrotoxicity incidence among patients with hematologic malignancies who frequently require antifungal treatment, to evaluate risk factors in patients who developed nephrotoxicity, and to discuss the utility of this agent providing similar efficacy at a lower cost compared to other agents in properly selected patients.

\section{Materials And Methods}

A total of 110 patients with hematologic malignancies who were admitted to the Adult Hematology Unit between January 2014 and November 2017 and who received intensive systemic chemotherapy and required anti-fungal treatment during that period were included in this study. Patients with AmB-d associated nephrotoxicity, epidemiologic data, clinical characteristics, and hematologic parameters were retrieved from the electronic database. Also, the following parameters were retrospectively assessed: demographic data such as age and gender, primary hematological diagnosis, chemotherapy regimen(s) administered, duration of expected neutropenia secondary to treatment, patient-related risk factors, concomitant nephrotoxic agents, laboratory parameters, creatinine (baseline, maximum, and most recent), stage of the renal failure, history of recurrent admission, indications, and duration of use for AmB-d, hepatotoxicity secondary to AmB-d use, metabolic abnormalities, discharge status, death, and transplantation.

Renal failure was staged according to AKIN (Acute Kidney Injury Network) criteria, which classifies acute kidney injury (AKI) in three stages of severity. (stages 1, 2, and 3) based on levels of serum creatinine and the patient's urine output. Accordingly, stage 1 injury was diagnosed with a $\geqslant 0.3 \mathrm{mg} / \mathrm{dl}$ or $1.5-2$ fold increase in baseline creatinine level (or urinary output for 6-12 hours $<0.5 \mathrm{ml} / \mathrm{kg} /$ hour); stage 2 injury was diagnosed with $>2-3$ fold increase in baseline serum creatinine (or $\geqslant 12$-hour urinary output $<0.5 \mathrm{ml} / \mathrm{kg} / \mathrm{hour}$ ); and stage 3 increase was diagnosed with $>3$-fold increase in baseline serum creatinine (or 24-hour urinary output $<0.3$ $\mathrm{ml} / \mathrm{kg}$ /hour or anuria $\geqslant 12$ hours). Hypokalemia was considered mild when serum potassium level was 3 to 3.4 meq/L, and severe when serum potassium level was $<3 \mathrm{meq} / \mathrm{L}$. The cut-off values for mild and severe hypomagnesemia were 1 to $1.7 \mathrm{mg} / \mathrm{dl}$, and $<1 \mathrm{mg} / \mathrm{dl}$, respectively.

Exclusion criteria were age $<18$ years, absence of AmB-d treatment, treatment on an outpatient basis, presence of kidney impairment at admission, known history of chronic renal disease, and missing data. The study was conducted in accordance with the Declaration of Helsinki and was approved by the Ethics Committee of the Medical Faculty of Bursa Uludağ University (February 19, 2020; no: 2020-3/1).

\section{Statistical analyses}

The normal distribution of the variables was assessed with the Shapiro-Wilk test. Presentable with normal distribution were expressed as mean \pm standard deviation, and comparison of two independent groups were performed using the independent sample t-test. Presentable without normal distribution were expressed as median values (minimum-maximum), and the two independent groups were compared with the MannWhitney U test. Categorical data were described with frequency and percentage (n,\%) and were compared with Pearson's chi-square test, Fisher's exact test, and Fisher-Freeman-Halton test. Statistical analyses were performed using the IBM SPSS Statistics 22.0 software pack (IBM Corp., Armonk, NY), at a significance level of $\mathrm{p}=0.05$.

\section{Results}

During the three-year study period, a total of 110 patients with hematological malignancies who were admitted to the Hematology Unit, Medical Faculty of Bursa Uludag University and treated with AmB-d as an anti-fungal regimen were recruited. Of these 110 patients, $62.7 \%(n=69)$ were male and $37.3 \%(n=41)$ were female, with an overall median age of $44 \pm 14.97$ years. Two groups of patients were defined on the basis of age $\leqslant 45$ years $(n=56,51.4 \%)$, and $>45$ years $(n=54,48.6 \%)$. The most common presentations included acute myeloid leukemia ( $n=53,48.2 \%$ ), acute lymphoid leukemia $(n=35,31.2 \%)$, and non-Hodgkin lymphoma $(\mathrm{n}=12,11 \%)$. Most cases of antifungal treatments were required in the course of intensive induction treatments such as $7+3$ remission-induction $(n=24,21.8 \%$; cytarabine, Idarubicin), Hyper-cvad ( $n=23$, 20.9\%; cyclophosphamide, vincristine, doxorubicin, cytarabine, methotrexate, and dexamethasone), and Flag-ida ( $n=20,18.2 \%$; fludarabine, cytarabine, idarubicine). In $87.3 \%$ of the patients $(n=96)$, the expected duration of neutropenia secondary to treatment was more than 10 days. In $60 \%$ of the patients ( $n=66$ ), steroids were administered, both as a part of the chemotherapeutic regimen and also as a premedication prior to AmB-d treatment. History of allogeneic transplantation was present in $10.9 \%$ of the patients $(n=12)$, while $64.5 \%(n=71)$ had a history of at least two hospital admissions. Of the recurrent admissions, 50\% 


\section{Cureus}

$(n=55)$ had occurred within the past six months. Among patients requiring antifungal treatment, $31.8 \%$

$(n=35)$ had at least one comorbid disease such as diabetes, hypertension, obesity, coronary artery disease, or hepatitis carrier condition (Table 1 ).

\begin{tabular}{|c|c|c|c|}
\hline \multicolumn{2}{|l|}{ Gender } & \multicolumn{2}{|l|}{ Median age $\%$ (age range) $44 \%$ (19-76) } \\
\hline Female & $37.3 \%(n=41)$ & Female & $\begin{array}{l}42.9 \% \\
(20- \\
69)\end{array}$ \\
\hline Male & $62.7 \%(n=69)$ & Male & $\begin{array}{l}44.7 \% \\
(19- \\
76)\end{array}$ \\
\hline \multicolumn{2}{|c|}{ Hematologic malignancy } & \multicolumn{2}{|l|}{ CTx protocol administered } \\
\hline AML & $48.2 \%(n=53)$ & $7+3$ remission induction (cytarabine $100 \mathrm{mg} / \mathrm{m}^{2}$ days $1-7 ;$ idarubicine $12 \mathrm{mg} / \mathrm{m}^{2}$ days $1-3$ ) & $\begin{array}{l}21.8 \% \\
(n=24)\end{array}$ \\
\hline ALL & $31.8 \%(n=35)$ & $\begin{array}{l}\text { Hyper-cvad (cyclophosphamide, } 300 \mathrm{mg} / \mathrm{m}^{2} \text { days } 1-3 \text {; vincristine, } 2 \mathrm{mg} \text { days } 4 \text { and } 11 \text {; doxorubicin } 50 \\
\mathrm{mg} / \mathrm{m}^{2} \text { day } 4 \text {; and dexamethasone } 40 \mathrm{mg} \text { days } 1-4 \text { and } 11-14 \text {. Courses } 2,4,6 \text {, and } 8 \text { consisted of } \\
\text { methotrexate, } 1 \mathrm{~g} / \mathrm{m}^{2} \text { day } 1 \text {; and cytarabine, } 3 \mathrm{~g} / \mathrm{m}^{2} \text { days } 2 \text { and } 3 \text { ) }\end{array}$ & $\begin{array}{l}20.9 \% \\
(n=23)\end{array}$ \\
\hline NHL & $11 \%(n=12)$ & FLAG-ida (fludarabine $30 \mathrm{mg} / \mathrm{m}^{2}$ days $1-5$; cytarabine $2 \mathrm{~g} / \mathrm{m}^{2}$ days $1-5$; idarubicin $10 \mathrm{mg} / \mathrm{m}^{2}$ days $1-3$ ) & $\begin{array}{l}18.2 \% \\
(n=20)\end{array}$ \\
\hline MDS & $1.8 \%(n=2)$ & $\begin{array}{l}\text { EMA (mitoxantrone } 12 \mathrm{mg} / \mathrm{m}^{2} \text { days } 1-3 \text {; etoposide } 200 \mathrm{mg} / \mathrm{m}^{2} \text { days } 8-10 \text { and cytarabine } 500 \mathrm{mg} / \mathrm{m}^{2} \\
\text { days } 1-3 \text { and } 8-10 \text { ) }\end{array}$ & $\begin{array}{l}12.8 \% \\
(n=14)\end{array}$ \\
\hline CLL & $1.8 \%(n=2)$ & Consolidation (cytarabine $6 \mathrm{~g} / \mathrm{m}^{2}$ days $1,3,5$ ) & $\begin{array}{l}11.8 \% \\
(n=13)\end{array}$ \\
\hline $\mathrm{HCL}$ & $1.8 \%(n=2)$ & Azacitidine $\left(50 \mathrm{mg} / \mathrm{m}^{2}\right.$ days $1-10$ or $75 \mathrm{mg} / \mathrm{m}^{2}$ days $\left.1-7\right)$ & $\begin{array}{l}1.8 \% \\
(n=2)\end{array}$ \\
\hline MM & $0.9 \%(n=1)$ & $\begin{array}{l}\text { DHAP (dexamethasone } 40 \mathrm{mg} \text { days } 1-4 \text {, cytarabine } 2000 \mathrm{mg} / \mathrm{m}^{2} / 12 \text { hours day } 2 \text {, cisplatin } 100 \\
\mathrm{mg} / \mathrm{m}^{2} \text { day } 1 \text { ) }\end{array}$ & $\begin{array}{l}1.8 \% \\
(n=2)\end{array}$ \\
\hline CML & $0.9 \%(n=1)$ & $\begin{array}{l}\text { R-FC (rituximab } 375-500 \mathrm{mg} / \mathrm{m}^{2} \text { day } 1 \text {; fludarabine } 25 \mathrm{mg} / \mathrm{m}^{2} \text { days } 1-3 \text { and cyclophosphamide } 250 \\
\mathrm{mg} / \mathrm{m}^{2} \text { days } 1-3 \text { ) }\end{array}$ & $\begin{array}{l}0.9 \% \\
(n=1)\end{array}$ \\
\hline AA & $0.9 \%(n=1)$ & Cladribine (0.14 mg/kg days $1-5)$ & $\begin{array}{l}0.9 \% \\
(n=1)\end{array}$ \\
\hline HL & $0.9 \%(n=1)$ & Other regimens & $\begin{array}{l}5.5 \% \\
(n=6)\end{array}$ \\
\hline & & Patients without CTx & $\begin{array}{l}3.6 \% \\
(n=4)\end{array}$ \\
\hline Transplan & & Steroid use & \\
\hline Yes & $10.9 \%(n=12)$ & Yes & $\begin{array}{l}60 \% \\
(n=66)\end{array}$ \\
\hline No & $89.1 \%(n=98)$ & No & $\begin{array}{l}40 \% \\
(n=44)\end{array}$ \\
\hline \multicolumn{2}{|c|}{ History of admission } & \multicolumn{2}{|l|}{ History of recurrent admission } \\
\hline First & $35.5 \%(n=39)$ & Within $<6$ months & $\begin{array}{l}50 \% \\
(n=55)\end{array}$ \\
\hline Second & $15.4 \%(n=17)$ & $>6$ months & $\begin{array}{l}14.5 \% \\
(n=16)\end{array}$ \\
\hline $\begin{array}{l}\text { Third or } \\
\text { more }\end{array}$ & $49.1 \%(n=54)$ & & \\
\hline $\begin{array}{l}\text { Expected } \\
\text { neutropen }\end{array}$ & $\begin{array}{l}\text { n of } \\
\text { sociation }\end{array}$ & isk facto & \\
\hline
\end{tabular}




\section{Cureus}

with the chemotherapy

regimen

$>10$ days $\quad 87.3 \%(n=96) \quad$ At least one

$<10$ days $\quad 12.7 \%(n=14) \quad$ None

\section{TABLE 1: Patient characteristics}

AA: aplastic anemia, AML: acute myeloid leukemia, ALL: acute lymphoblastic leukemia, NHL: non-Hodgkin lymphoma, MDS: myelodysplastic syndrome, CLL: chronic lymphocytic leukemia, HCL: hairy cell leukemia, MM: multiple myeloma, CML: chronic myeloid leukemia, HL: Hodgkin lymphoma.

Indications for AmB-d use included empiric treatment (fever, neutropenia, or other causes) in 56.4\% ( $\mathrm{n}=62$ ), documented fungal infection (microbial growth in blood, urine, tissue, central nervous system, sputum, or other cultures) in $19.1 \%$ ( $n=21$ ), and presence of radiological findings (thorax CT image suggestive of invasive pulmonary Aspergillosis) in 24.5\% ( $n=27)$.

The mean weight of patients was $74.1 \pm 13.32 \mathrm{~kg}$ (min: 40, max:112 kg), and the mean body surface area was $1.81 \pm 0.18 \mathrm{~m}^{2}$ (min: 1.4, max: $2.2 \mathrm{~m} 2$ ). AmB-d infusions were administered over a two to six-hour period. The median daily dose was $70 \mathrm{mg}$ (40-100; mean $71.9 \pm 13.03 \mathrm{mg}$ ), with a cumulative dose of $480 \mathrm{mg}$ (502250 ; mean $712.0 \pm 582.3)$. The median duration of AmB-d treatment was seven days (1-45 days; mean 10.5 \pm 9.35 days). A cumulative dose exceeding $1 \mathrm{~g}$ was given in $28.2 \%(\mathrm{n}=31)$ of the patients. Duration, daily dose, and cumulative dose of AmB-d treatment were not significantly different between sexes $(p>0.05)$. On the other hand, the duration and cumulative dose of AmB-d treatment were significantly higher among those $\leqslant 45$ years of age $(\mathrm{p}<0.05)$. The age groups were comparable with respect to daily AmB-d dose.

The laboratory parameters at the time of admission were as follows: white blood cell (WBC) $7.6 \times 10^{3} / \mu \mathrm{L}$ (min 0.1 - max 662), neutrophil (neu) $2.6 \times 10^{3} / \mu \mathrm{L}$ (min $0.01-\max 68.9$ ), hemogobin (hgb) $10.2 \pm 2.2 \mathrm{~g} / \mathrm{dl}$, platelets (plt) $62.2 \times 10^{3} / \mu \mathrm{L}(\min 4.2-\max 570)$, urea: $30 \mathrm{mg} / \mathrm{dl}(\min 9-\max 95)$, creatinine $0.7 \mathrm{mg} / \mathrm{dl}(\min 0.4-\max$ 3.8), sodium $138 \mathrm{mmol} / \mathrm{L}$ (min 122 - max 144), potassium $3.9 \mathrm{meq} / \mathrm{L}$ ( $\min 2$ - max 5.7), calcium $8.5 \mathrm{mg} / \mathrm{dl}$ (min 7.2- max 10.4), aspartate aminotransferase (AST) $28 \mathrm{U} / \mathrm{L}$ ( $\min 7-\max 194)$, alanine aminotransferase (ALT) 26.5 U/L (min 6-max 315), and lactic dehydrogenase (LDH) 316.5 U/L (min 94- max 4500). Pre-treatment baseline creatinine was $0.69 \pm 0.13 \mathrm{mg} / \mathrm{dl}$ (median 0.7, min: 0.4-max:1.08), and post-treatment final creatinine was $0.9 \pm 0.4 \mathrm{mg} / \mathrm{dl}$ (median 0.8; min: 0.4-max: 2.24), with highest creatinine level of $1.01 \pm 0.42$ $\mathrm{mg} / \mathrm{dl}$ (median 0.9; min: 0.5-max:2.25) recorded during treatment. While there were significant gender differences in terms of baseline and highest creatinine levels $(\mathrm{p}<0.01)$, the final post-treatment creatinine was similar across the sexes. Also, there were significant differences in terms of creatinine between age groups ( $\mathrm{p}<0.05$ ), while the final and maximum creatinine levels were comparable between the groups. Baseline and highest creatinine did not differ between patients with at least one risk factor (diabetes, hypertension, coronary artery disease, etc.) and patients with no risk factor (Table 2). 


\section{Cureus}

\begin{tabular}{|c|c|c|c|}
\hline & Basal creatinine & Final creatinine & Highest creatinine \\
\hline Overall & $0.69 \pm 0.13$ & $0.80(0.4-2.24)$ & $0.90(0.50-2.25)$ \\
\hline \multicolumn{4}{|l|}{ Gender } \\
\hline Female $(\mathrm{n}=41)$ & $0.64 \pm 0.12$ & $0.70(0.40-2.04)$ & $0.70(0.50-2.04)$ \\
\hline Male $(n=69)$ & $0.72 \pm 0.13$ & $0.86(0.46-2.24)$ & $1.00(0.54-2.25)$ \\
\hline P-value & 0.001 & 0.008 & 0.001 \\
\hline \multicolumn{4}{|l|}{ Age } \\
\hline$\leq 45(n=56)$ & $0.66 \pm 0.13$ & $0.70(0.40-2.24)$ & $0.90(0.53-2.25)$ \\
\hline$>45(n=54)$ & $0.72 \pm 0.13$ & $0.82(0.46-2.04)$ & $0.90(0.50-2.04)$ \\
\hline P-value & 0.018 & 0.107 & 0.917 \\
\hline \multicolumn{4}{|l|}{ Risk factors } \\
\hline Absent $(\mathrm{n}=75)$ & $0.69 \pm 0.12$ & $0.80(0.43-2.24)$ & $0.86(0.50-2.25)$ \\
\hline Present $(n=35)$ & $0.69 \pm 0.15$ & $0.81(0.40-2.04)$ & $0.92(0.53-2.12)$ \\
\hline P-value & 0.968 & 0.345 & 0.40 \\
\hline
\end{tabular}

TABLE 2: Amphotericin B nephrotoxicity according to gender, age, and risk factors

Of the 110 patients included, no AmB-d nephrotoxictiy was found in $63.6 \%(n=70)$, while renal failure was observed during treatment in $36.4 \%(n=40)$. Of these latter group of patients, $75 \%(n=30)$ were male. Stages 1,2 , and 3 renal failure were found in $20.9 \%(n=23), 11.8 \%(n=13)$, and $3.7 \%(n=4)$ of the patients, respectively (Table 3). Among patients with renal failure, the cumulative dose of AmB- $\mathrm{d}$ was $<0.5 \mathrm{~g}$ in $57.5 \%$, 0.5 to $1 \mathrm{~g}$ in $17.5 \%(\mathrm{n}=7), 1$ to $1.5 \mathrm{~g}$ in $12.5 \%(\mathrm{n}=5), 1.5$ to $2 \mathrm{~g}$ in $7.5 \%(\mathrm{n}=3)$, and $>2 \mathrm{~g}$ in $5 \%(\mathrm{n}=2)$. The average time to development of nephrotoxicity after initiation of AmB-d treatment was four days (min: 2, max: 31). 


\section{Cureus}

\begin{tabular}{|c|c|c|c|c|}
\hline Acute renal failure & Total & Female & Male & \\
\hline Absent & $63.6 \%(n=70)$ & $75.6 \%(n=31)$ & $56.5 \%(n=39)$ & \multirow{5}{*}{$P=0.098$} \\
\hline Present & $36.4 \%(n=40)$ & $24.4 \%(n=10)$ & $43.5 \%(n=30)$ & \\
\hline Stage I & $20.9 \%(n=23)$ & $9.8 \%(n=4)$ & $27.5 \%(n=19)$ & \\
\hline Stage II & $11.8 \%(n=13)$ & $9.8 \%(n=4)$ & $13.1 \%(n=9)$ & \\
\hline Stage III & $3.7 \%(n=4)$ & $4.8 \%(n=2)$ & $2.9 \%(n=2)$ & \\
\hline \multicolumn{5}{|l|}{ Hypomagnesemia } \\
\hline Absent & $41.8 \%(n=46)$ & $36.6 \%(n=15)$ & $44.9 \%(n=31)$ & \multirow{4}{*}{$P=0.15$} \\
\hline Present & $58.2 \%(n=64)$ & $63.4 \%(n=26)$ & $55.1 \%(n=38)$ & \\
\hline$<1 \mathrm{mg}$ Severe & $1.8 \%(n=2)$ & $4.9 \%(n=2)$ & $0 \%(n=0)$ & \\
\hline 1-1.7 mg Mild & $56.4 \%(n=62)$ & $58.5 \%(n=24)$ & $55.1 \%(n=38)$ & \\
\hline \multicolumn{5}{|l|}{ Hypokalemia } \\
\hline Absent & $30 \%(n=33)$ & $24.4 \%(n=10)$ & $33.3 \%(n=23)$ & \multirow{4}{*}{$P=0.306$} \\
\hline Present & $70 \%(n=77)$ & $75.6 \%(n=31)$ & $66.7 \%(n=46)$ & \\
\hline$<3$ severe & $37.3 \%(n=41)$ & $46.3 \%(n=19)$ & $31.9 \%(n=22)$ & \\
\hline 3-3.4 mild & $32.7 \%(n=36)$ & $29.3 \%(n=12)$ & $34.8 \%(n=24)$ & \\
\hline \multicolumn{5}{|l|}{ Hepatotoxicity } \\
\hline Absent & $89 \%(n=87)$ & $88.1 \%(n=32)$ & $79.7 \%(n=55)$ & \multirow{2}{*}{$P=1.00$} \\
\hline Present & $21 \%(n=23)$ & $21.9 \%(n=9)$ & $20.3 \%(n=14$ & \\
\hline
\end{tabular}

\section{TABLE 3: Side effects associated with AmB-d treatment}

All patients received at least one potential nephrotoxic drug during the anti-fungal treatment, including acyclovir, methotrexate, vancomycin, loop diuretics (furosemide), and aminoglycosides (amikacin).

The reasons for discontinuation of AmB-d therapy included completion of treatment, patient discharge or death in $42.7 \%(n=47)$, allergic reactions in $21 \%(n=23)$, development of renal failure in $19.1 \%(n=21)$, switching to another effective treatment in $9.1 \%(\mathrm{n}=10)$, hepatotoxicity in $5.5 \%(\mathrm{n}=6)$, and severe hypokalemia resistant to replacement treatment in $2.7 \%(\mathrm{n}=3)$.

Table 4 shows the distribution of renal failure patients according to risk factors, chemotherapy protocol, history of recurrent admissions, history of transplantation, and steroid use. Based on these models, none of these variables were significantly associated with the risk of AmB-d-related nephrotoxicity. 


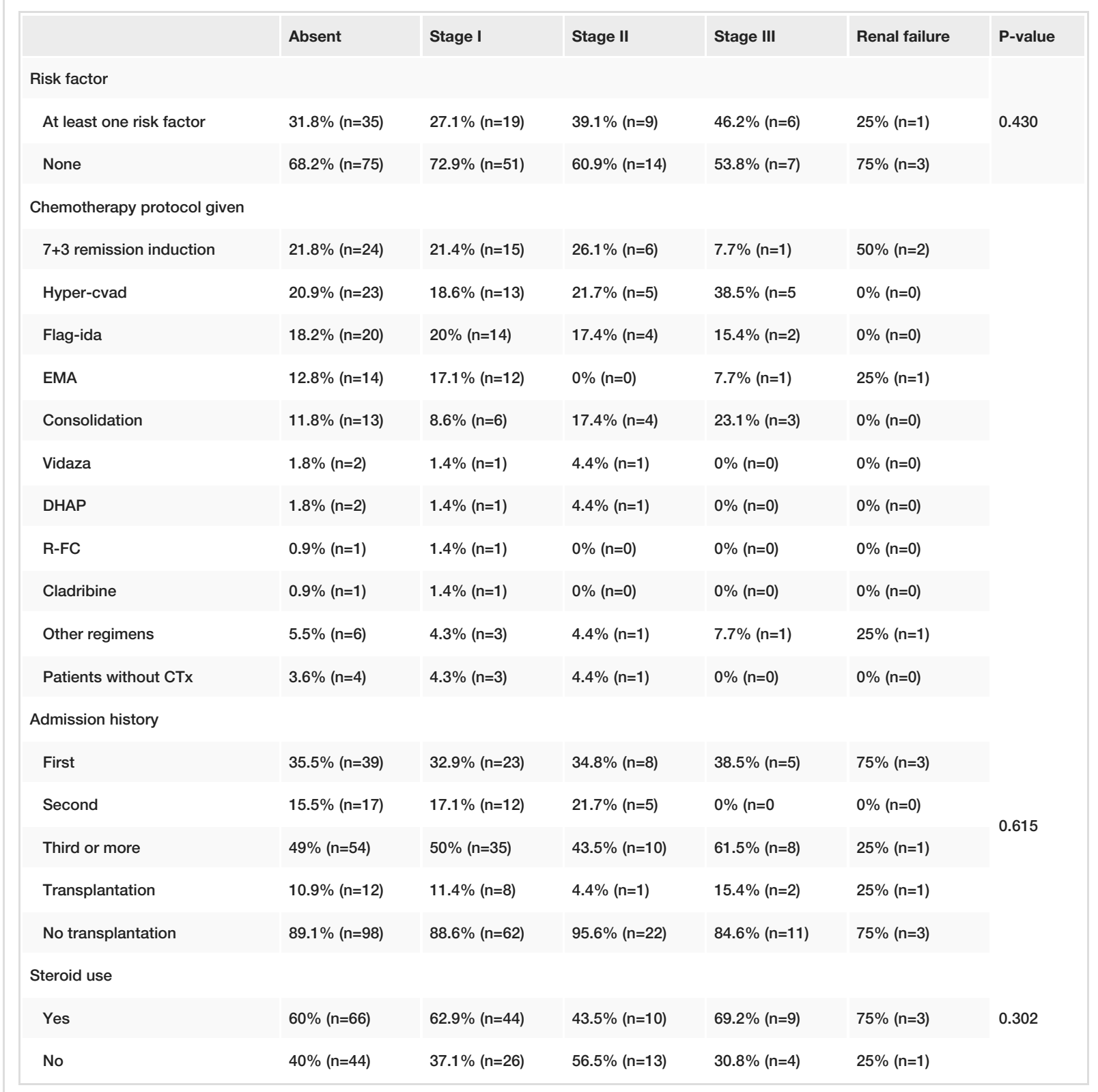

TABLE 4: Comparisons regarding renal failure

In $52.5 \%(\mathrm{n}=21)$ of the 40 patients who developed nephrotoxicity, the treatment was discontinued due to nephrotoxicity, while the reasons for treatment discontinuation in the remaining 19 patients were hepatotoxicity in one, switch to other effective treatments in two, discharge in seven, switch to oral therapy in two, adequate treatment of the causative agent in three, and death in four. In this patient group, the mean duration of medication use was seven days (2-45).

Toxicities other than AmB-d-related renal failure were also assessed. Among these, the most common toxic effects included hypokalemia, hypomagnesemia, and hepatotoxicity. In $70 \%(n=77)$ of the 110 patients, hypokalemia was detected, which was severe (potassium $<3 \mathrm{mEq})$ in $37.3 \%(n=41)$ requiring potassium replacement. Hypomagnesemia was found in $58.2 \%(n=64)$ of the patients, with only two patients experiencing severe hypomagnesemia $(<1 \mathrm{mEq})$. On the other hand, $21 \%(\mathrm{n}=23)$ of the patients had hepatotoxicity (Table 3), being grade I (AST/ALT $\leqslant 2.5 \times \mathrm{N}$, bil:N) in seven, grade II (AST/ALT: $2.6-5 \times \mathrm{N}$, bil: $<1.5 \times \mathrm{N}$ ) in six, grade III (AST/ALT: 5.1-20 x N, bil:1.5 $-3 \times \mathrm{N}$ ) in seven, and grade IV (AST/ALT: >20 x N, bil: $>3 \times \mathrm{N}$ ) in three.

Of the 110 patients, $56.4 \%(n=62)$ were discharged, while $43.6 \%(n=48)$ died. The mortality rate was $38.6 \%$ among those who did not develop nephrotoxicity during AmB-d treatment, while $52.5 \%$ among those who developed AmB-d associated nephrotoxicity. 


\section{Discussion}

In recent years, $A m B-d$ has fallen into disfavor in the treatment of fungal infections due to its untoward effects. In this regard, nephrotoxicity is the most important dose-limiting side effect associated with AmB-d use. Although the exact mechanisms of AmB-d associated nephrotoxicity remain obscure, studies suggest that renal vasoconstriction and tubular injury may play a major role $[6,10,11]$. The tubular injury also leads to hypokalemia and hypomagnesemia $[6,10,11]$. In a study by Walsh et al. comparing AmB-d and liposomal $\mathrm{AmB}$, both drugs were associated with similar survival rates ( $93 \%$ vs. $90 \%$ ) and less responses (58\%, for each), while toxicities were less frequent in the liposomal AmB arm [8]. In another meta-analysis, the incidence of AmB-d-associated nephrotoxicity was higher (32.5\% vs. $14.5 \%$ ) [8,12]. However, lipid-based formulations and newer generation anti-fungal agents are associated with a higher economic burden due to their high acquisition costs. In one study, Roux et al. reported that most anti-fungal treatments for invasive candidiasis administered in patients admitted to intensive care units may actually be unnecessary, leading to increased treatment costs. Therefore, these authors recommended the use of AmB- $d$ for the first-line treatment of such infections, based on similar efficacy with newer antifungal agents and lower treatment costs [13]. While lipid-based formulations have largely replaced AmB- $d$ in countries with adequate resources, the conventional formulation continues to be widely utilized in developing countries, where cost-related issues remain a source of concern. However, when the new conditions of the world combined with the major burden of the pandemic on healthcare systems are taken into consideration, it is likely that cost will play a more important role in treatment decisions throughout the world, not only in the developing countries. Similarly, although the use of AmB-d is declining, it still remains a therapeutic option in certain indications in our country.

A generally reversible and temporary decrease in GFR has been reported in approximately $5 \%$ to $80 \%$ of patients receiving treatment with AmB-d [14,15]. The net effect involves an elevation of serum creatinine levels. In a nine-year-long retrospective study by Harbarth et al. involving 494 patients, nephrotoxicity of any degree was reported in 138 (28\%) during AmB-d treatment, with 58 patients (12\%) having moderately severe nephrotoxicity [14]. In another report from the Hematology-Oncology and Stem Cell Transplantation Unit of the Talegani Hospital, Tehran increased serum creatinine and BUN was reported in 9 of 35 patients (25.7\%) during AmB-d treatment [15]. Khalili et al. observed acute kidney injury in 10 of 13 patients (76.92\%) who were treated with AmB-d alone; however, the incidence of nephrotoxicity was $86.6 \%$ among those who also received ceftriaxone and/or vancomycin in combination with AmB-d [16]. In our study, the incidence of nephrotoxicity was evaluated in patients who only received the AmB-d formulation, and this figure was found to be $36.4 \%(n=40)$. Of these cases, $11.8 \%(n=13)$ and $3.7 \%(n=4)$ had stage II and III nephrotoxicity, respectively. The wide variation in the reported incidence of AmB-d-related nephrotoxicity may be due to differences in methodologies, clinical settings, related risk factors, and definitions used. The lower rates of nephrotoxicity in some studies may be related to the use of liposomal AmB, while higher rates in our study may be explained on the basis of the use of the conventional AmB only in our patient group.

Stage 3 kidney failure exclusively associated with the use of conventional AmB-d among patients with hematological malignancies is rare, and renal dysfunction is multifactorial in most cases. Other potential culprits include the extent of the fungal infection, the current status of the underlying hematologic malignancy, presence/absence of comorbid conditions, use of other potentially nephrotoxic agents, involvement of other infectious pathogens, sepsis, and septic shock. Potential risk factors identified in previous prospective or retrospective studies include male gender, an average daily AmB-d dose of $35 \mathrm{mg}$, cumulative dose of AmB-d exceeding 2 to $5 \mathrm{~g}$, dehydration, concomitant use of diuretics or nephrotoxic agents (e.g., aminoglycosides, cyclosporine, foscarnet, cisplatin, and ifosfamide) or corticosteroids, and presence of kidney dysfunction at baseline [17-19]. It is generally recommended to avoid AmB-d treatment in the presence of two or more of the above-listed risk factors [14,20]. The reported incidence of nephrotoxicity in patients receiving two or more nephrotoxic agents is $41 \%$ [8]. AmB-d related nephrotoxicity is generally reversible upon discontinuation of treatment [21,22], although kidney dysfunction may recur if treatment is re-initiated [22]. Renal replacement therapy such as dialysis may be required in approximately $15 \%$ of the patients affected [20,23]. In the current study, AmB-d related nephrotoxicity improved without treatment in $47.5 \%$ of the affected patients, and no patients required dialysis. While some of our patients developed stage 1-2 renal failure, these subjects tolerated the treatment under close supervision and without discontinuation of AmB-d, and laboratory parameters did not worsen, and the event got better over time. Laboratory parameters were checked on a daily basis, and prompt treatment adjustments were done when required. Therefore, mild renal failure occurring after initiation of AmB-d may not always require treatment discontinuation. In younger patients with good performance status and no risk factors, a continuation of treatment may be considered, provided that the patient is closely monitored. Adverse events related to drugs represent a significant cause of morbidity and mortality in neutropenic patients with hematologic malignancies. In our study, toxicities other than nephrotoxicity were also related with regard to their association with AmB-d treatment. Among these, the most common ones included drug infusion reactions (21\%), hypokalemia (70\%), hypomagnesemia (58.2\%), and hepatotoxicity (21\%). Most of the infusion reactions (86\%) occur in the first five minutes of infusion [6]. In a study of liposomal AmB-d, Roden et al. reported that almost more than $20 \%$ of their patients had infusion-related reactions [24]. Despite some studies reporting higher rates of infusion reactions with the use of AmB-d versus the liposomal formulation (52\% vs. $21 \%$ ) [8], we failed to observe such differences. Only patients using AmB-d were included in this study. No comparison was made with the liposomal formulation. The treatment 
was discontinued in patients who experienced severe infusion reactions despite premedication. Symptoms fully recovered with no persistent effects in all patients. Seventy percent of our patients had hypokalemia in association with the use of AmB-d, and this figure was similar to the reported incidence of $75 \%$ to $90 \%$ [25]. In a recent study from Iran examining liposomal $\mathrm{AmB}$ in hematology-oncology practice, the reported incidence was 45\% [26]. Hypomagnesemia, which is generally mild, is a frequent side effect of AmB treatment secondary to renal loss of magnesium. Therefore, routine monitoring of serum magnesium levels is recommended during AmB treatment [27]. In some publications, the reported incidence of hypomagnesemia ranged between $15 \%$ and $100 \%$, depending on the dose and formulation of AmB [28]. Severe hypokalemia and hypomagnesemia secondary to AmB use may lead to metabolic complications, rhabdomyolysis, and life-threatening arrhythmias [25]. Although hypokalemia and hypomagnesemia were not uncommon in our patient group, no life-threatening serious complications were observed. Patients were closely monitored with laboratory tests according to their clinical characteristics, and appropriate replacement therapy was given. AmB-d related hepatotoxicity is rarely reported in the literature [29]. Persat et al., in a group of 20 patients with leukemia, observed no hepatotoxicity with itraconazole alone, while in 11 of the 12 (92\%) leukemic patients receiving itraconazole combined with AmB-d had significant elevations in their liver enzymes [30]. In our study, $21 \%$ of the patients also had elevated liver enzymes, although only one patient required discontinuation of treatment due to hepatotoxicity. Our analysis based on clinical characteristics of the patients did not suggest a causal link between the use of AmB- $d$ and hepatotoxicity. It appears that the risk of hepatotoxicity is higher in the context of multi-drug therapy.

\section{Conclusions}

In conclusion, nephrotoxicity was observed in approximately one-third of our patients (36.4\%) during AmB$\mathrm{d}$ therapy, which resolved spontaneously without specific intervention in almost half of these subjects (47.5\%). The mortality rate in those with nephrotoxicity was higher than those without nephrotoxicity, although this finding should be interpreted with caution due to the retrospective design of our study precluding direct comparisons. Other potential factors associated with mortality include the extent of the fungal infection, underlying hematologic malignancy, presence of comorbid conditions, multi-drug therapy, involvement of other infectious pathogens, presence of sepsis or septic shock, and the need for treatment in frail and elderly patients. In this group of patients, it would be prudent to closely monitor indices of kidney functions (e.g., serum creatinine, urea, potassium, and magnesium) as well as liver enzymes, in addition to daily clinical assessments with vital signs and physical examination. Appropriate patient selection and expertise of the clinical team are the two most important determinants of a successful management plan. In our country and other developing countries, avoidance of AmB-d therapy on the basis of potential nephrotoxicity concerns in favor of costlier treatments may not represent an appropriate approach for a carefully selected group of patients. Therefore, we believe that AmB-d should remain a valid therapeutic option in these settings, provided that patients are carefully selected and effectively monitored.

\section{Additional Information \\ Disclosures}

Human subjects: Consent was obtained or waived by all participants in this study. Ethics Committee of the Medical Faculty of Bursa Uludağ University issued approval 2020-3/1. Animal subjects: All authors have confirmed that this study did not involve animal subjects or tissue. Conflicts of interest: In compliance with the ICMJE uniform disclosure form, all authors declare the following: Payment/services info: All authors have declared that no financial support was received from any organization for the submitted work. Financial relationships: All authors have declared that they have no financial relationships at present or within the previous three years with any organizations that might have an interest in the submitted work. Other relationships: All authors have declared that there are no other relationships or activities that could appear to have influenced the submitted work.

\section{References}

1. Johnson RH, Einstein HE: Amphotericin B and coccidioidomycosis. Ann N Y Acad Sci. 2007, 1111:434-41. 10.1196/annals. 1406.019

2. Gibbs WJ, Drew RH, Perfect JR: Liposomal amphotericin B: clinical experience and perspectives . Expert Rev Anti Infect Ther. 2005, 3:167-81. 10.1586/14787210.3.2.167

3. Ostrosky-Zeichner L, Marr KA, Rex JH, Cohen SH: Amphotericin B: time for a new" gold standard" . Clin Infect Dis. 2003, 415:25.

4. Warnock DW: Amphotericin B: an introduction. J Antimicrob Chemother. 1991, 28 Suppl B:27-38. 10.1093/jac/28.suppl b.27

5. Anaissie EJ, Darouiche RO, Abi-Said D, et al.: Management of invasive candidal infections: results of a prospective, randomized, multicenter study of fluconazole versus amphotericin B and review of the literature. Clin Infect Dis. 1996, 23:964-72. 10.1093/clinids/23.5.964

6. Laniado-Laborín R, Cabrales-Vargas MN: Amphotericin B: side effects and toxicity. Rev Iberoam Micol. 2009, 26:223-7. 10.1016/j.riam.2009.06.003

7. Karimzadeh I, Khalili H, Farsaei S, Dashti-Khavidaki S, Sagheb MM: Role of diuretics and lipid formulations in the prevention of amphotericin B-induced nephrotoxicity. Eur J Clin Pharmacol. 2013, 69:1351-68. 10.1007/s00228-013-1472-1

8. Walsh TJ, Finberg RW, Arndt C, et al.: Liposomal amphotericin B for empirical therapy in patients with 
persistent fever and neutropenia. National Institute of Allergy and Infectious Diseases Mycoses Study Group. N Engl J Med. 1999, 340:764-71. 10.1056/NEJM199903113401004

9. Cagnoni PJ: Liposomal amphotericin B versus conventional amphotericin B in the empirical treatment of persistently febrile neutropenic patients. J Antimicrob Chemother. 2002, 49 Suppl 1:81-6. 10.1093/jac/49.suppl_1.81

10. Deray G: Amphotericin B nephrotoxicity. J Antimicrob Chemother. 2002, 49 Suppl 1:37-41. 10.1093/jac/49.suppl_1.37

11. Zager RA, Bredl CR, Schimpf BA: Direct amphotericin B-mediated tubular toxicity: assessments of selected cytoprotective agents. Kidney Int. 1992, 41:1588-94. 10.1038/ki.1992.229

12. Mistro S, Maciel Ide M, de Menezes RG, Maia ZP, Schooley RT, Badaró R: Does lipid emulsion reduce amphotericin B nephrotoxicity? A systematic review and meta-analysis. Clin Infect Dis. 2012, 54:1774-7. 10.1093/cid/cis290

13. Roux D, Gaudry S, Dreyfuss D: Conventional amphotericin B should remain in the armamentarium as a cheap and efficient waiting solution. Intensive Care Med. 2020, 46:558-9. 10.1007/s00134-019-05782-z

14. Harbarth S, Pestotnik SL, Lloyd JF, Burke JP, Samore MH: The epidemiology of nephrotoxicity associated with conventional amphotericin B therapy. The. Am J Med. 2001, 111:528-34. 10.1016/S00029343(01)00928-7

15. Tavakoli-Ardakani M, Eshraghi A, Hajhossein Talasaz A, Salamzadeh J: A drug utilization evaluation study of amphotericin B in neutropenic patients in a teaching hospital in Iran. Iran J Pharm Res. 2012, 11:151-6.

16. Khalili H, Bairami S, Kargar M: Antibiotics induced acute kidney injury: incidence, risk factors, onset time and outcome. Acta Medica Iranica. 2013, 51:871-8.

17. Fisher MA, Talbot GH, Maislin G, McKeon BP, Tynan KP, Strom BL: Risk factors for amphotericin Bassociated nephrotoxicity. Am J Med. 1989, 87:547-52. 10.1016/S0002-9343(89)80612-6

18. Luber AD, Maa L, Lam M, Guglielmo BJ: Risk factors for amphotericin B-induced nephrotoxicity. J Antimicrob Chemother. 1999, 43:267-71. 10.1093/jac/43.2.267

19. Goldman RD, Ong M, Wolpin J, Doyle J, Parshuram C, Koren G: Pharmacological risk factors for amphotericin B nephrotoxicity in children. J Clin Pharmacol. 2007, 47:1049-55.

20. Bates DW, Su L, Yu DT, Chertow GM, Seger DL, Gomes DR, Platt R: Correlates of acute renal failure in patients receiving parenteral amphotericin B. Kidney Int. 2001, 60:1452-9. 10.1046/j.15231755.2001.00948.x

21. Heidemann HT, Gerkens JF, Spickard WA, Jackson EK, Branch RA: Amphotericin B nephrotoxicity in humans decreased by salt repletion. Am J Med. 1983, 75:476-81. 10.1016/0002-9343(83)90353-4

22. Sacks P, Fellner SK: Recurrent reversible acute renal failure from amphotericin . Arch Int Med. 1987, 147:593-5. 10.1001/archinte.1987.00370030191038

23. Karimzadeh I, Farsaei S, Khalili H, Dashti-Khavidaki S: Are salt loading and prolonging infusion period effective in prevention of amphotericin B-induced nephrotoxicity?. Expert Opin Drug Saf. 2012, 11:969-83. 10.1517/14740338.2012.721775

24. Roden MM, Nelson LD, Knudsen TA, et al.: Triad of acute infusion-related reactions associated with liposomal amphotericin B: analysis of clinical and epidemiological characteristics. Clin Infect Dis. 2003, 36:1213-20. 10.1086/374553

25. Wazny LD, Brophy DF: Amiloride for the prevention of amphotericin B-induced hypokalemia and hypomagnesemia. Ann Pharmacother. 2000, 34:94-7. 10.1345/aph.19127

26. Karimzadeh I, Heydari M, Ramzi M, Sagheb MM: Frequency and associated factors of amphotericin b nephrotoxicity in hospitalized patients in hematology-oncology wards in the Southwest of Iran. Nephrourol Mon. 2016, 8:e39581. 10.5812/numonthly.39581

27. Barton C, Pahl M, Vaziri N, Cesario T: Renal magnesium wasting associated with amphotericin B therapy . Am J Med. 1984, 77:471-4. 10.1016/0002-9343(84)90106-2

28. Atsmon J, Dolev E: Drug-induced hypomagnesaemia: scope and management. Drug Saf. 2005, 28:763-88. 10.2165/00002018-200528090-00003

29. Gill J, Sprenger HR, Ralph ED, Sharpe MD: Hepatotoxicity possibly caused by amphotericin B . Ann Pharmacother. 1999, 33:683-5. 10.1345/aph.18181

30. Persat F, Schwartzbrod PE, Troncy J, Timour Q, Maul A, Piens MA, Picot S: Abnormalities in liver enzymes during simultaneous therapy with itraconazole and amphotericin B in leukaemic patients. J Antimicrob Chemother. 2000, 45:928-9. 10.1093/jac/45.6.928 